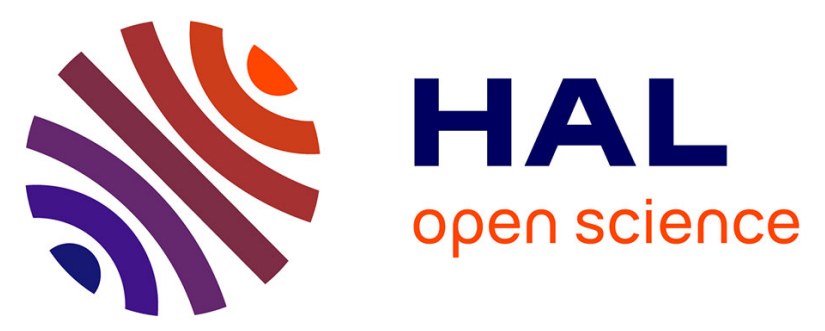

\title{
Acceptance and Organizational Aspects of Oral tele-Consultation: a French Study
}

Roland Petcu, Roxana Ologeanu-Taddei, Isabelle Bourdon, Chris Kimble, Nicolas Giraudeau

\section{- To cite this version:}

Roland Petcu, Roxana Ologeanu-Taddei, Isabelle Bourdon, Chris Kimble, Nicolas Giraudeau. Acceptance and Organizational Aspects of Oral tele-Consultation: a French Study. Hawaii International Conference on System Sciences, The Institute of Electrical and Electronics Engineers, Jan 2016, Hawaii United States. pp.3124-3132. halshs-01273672

\section{HAL Id: halshs-01273672 \\ https://shs.hal.science/halshs-01273672}

Submitted on 12 Feb 2016

HAL is a multi-disciplinary open access archive for the deposit and dissemination of scientific research documents, whether they are published or not. The documents may come from teaching and research institutions in France or abroad, or from public or private research centers.
L'archive ouverte pluridisciplinaire HAL, est destinée au dépôt et à la diffusion de documents scientifiques de niveau recherche, publiés ou non, émanant des établissements d'enseignement et de recherche français ou étrangers, des laboratoires publics ou privés. 


\section{Acceptance and Organizational Aspects of Oral tele-Consultation: a French Study}

\author{
Roland Petcu \\ Montpellier Research in \\ Management \\ Montpellier University \\ France \\ roland.petcu@gmail.com
}

Roxana Ologeanu-Taddei

Montpellier Research in

Management

Montpellier University

France

roxana.ologeanu-taddei@univ-montp2.fr

\author{
Isabelle Bourdon \\ Montpellier Research in \\ Management \\ Montpellier University \\ France \\ isabelle.bourdon.pro@gmail.com
}

\author{
Chris Kimble \\ KEDGE Business School Marseille \\ Montpellier Research in Management \\ France \\ chris.kimble@kedgebs.com
}

\author{
Nicolas Giraudeau \\ Montpellier University \\ University Hospital of Montpellier \\ France \\ nicolas.giraudeau@umontpellier.fr
}

\begin{abstract}
This is a descriptive article focusing on the eDENT project, the first oral tele-consultation in France; an experimentation of asynchronous dental tele-consultations meant to improve access to dental care for people who have limited access to regular consultations with a dentist such as dependent elderly, disabled people or prisoners. We consider that the generalization of dental tele-consultations is related to the acceptance of the e-DENT system, as stated by Broens \& et al. (2007). This paper proposes a new methodological approach for assessing the perception of a medical procedure, involving the use of technology, for subjects with minor, mild or severe cognitive impairment. Semi-structured interviews, indirect patient observations and non-participant observations have allowed us to highlight the nurses' acceptance of the e-DENT system, the patients' perception of the oral tele-consultation procedure and to describe the induced organizational changes.
\end{abstract}

\section{Introduction}

The prevalence of age-related health problems is an important public health concern related to the growing proportion of older individuals in the general population worldwide. Projections show that by 2050 the number of individuals older than 60 will be approximately 2 billion and will account for $22 \%$ of the world's population. It is also estimated that 35.6 million people are currently living with dementia worldwide and that the number will almost double every 20 years, reaching 115.4 million in 2050 [16]. In
"Aging Facts", the World Health Organization considers as particularly important the fact that "the number of older people who are no longer able to look after themselves in developing countries is forecast to quadruple by 2050 . Many of the very old lose their ability to live independently because of limited mobility, frailty or other physical or mental health problems. Many require some form of long-term care, which can include home nursing, community care and assisted living, residential care and long stays in hospitals"[30].

In France, as of $1^{\text {st }}$ of January $2014,18 \%$ of the population is 65 or older, a proportion which has increased by 3.4 percent during the past 20 years. More than half of this population are individuals who are 75 or older [3].

In this paper we argue the need for new or better adapted methodological approaches in evaluating the elderly population's acceptance of procedures and technology that take into consideration their altered cognition. Our study will be conducted on a subpopulation of the elderly, namely those in long term care facilities.

\subsection{The identified problem}

A report by the French Health Authority (Haute Autorité de Santé) on caries prevention strategies highlighted the fact that residents of health, medicalsocial and social-care facilities whether they present reduced mobility or not, have a very limited access to dental care. According to different studies, $86.2 \%$ of residents did not have a dental consultation in the past 12 months (Provence Alpes Côte d'Azur region, France) and $42 \%$ of residents did not have one for 
more than 5 years (Essonne department, France). At national level, $35 \%$ of dentate residents need conservative dentistry interventions, without mentioning other oral health needs.

This situation could be explained by the fact that nursing homes have a difficult time finding dentists willing to come into such institutions to make consultations and these institutions are often understaffed and oral hygiene is usually left last.

The first step towards improving the oral health of the patients would be to evaluate the current oral health status, to have a diagnosis and a treatment plan. Unfortunately, this approach raises another type of problem, namely transportation costs for the patients to the dentist's office or the transportation costs for the dentist to the healthcare facility, which also comes with limitations in terms of proper equipment and materials.

\section{2. e-DENT, an innovative approach}

In this context, the e-DENT project was designed to provide access to an oral consultation for those who do not have access to one, or for whom access is limited: dependent elderly, disabled people or prisoners. The project has been facilitated by a French law, introduced in 2010, which regulates the telemedicine practices. This law describes the legal framework of different tele-medicine acts, like: tele-consultation, teleexpertise, tele-monitoring, tele-assistance and teleregulation. The e-DENT project is related to teleconsultation in the dentistry field and consists in the asynchronous remote consultation of a patient assisted by a healthcare professional.

The e-DENT project, based in the LanguedocRoussillon area of Southern France, involves the Montpellier University Hospital and the company ORAL-B (Procter \& Gamble). The project started in 2014 and it is supported and financed by the Regional Health Agency of Languedoc-Roussillon for two years. The pilot will include a total of 800 subjects: 600 elderly people, 100 prisoners and 100 frail people from twelve long-term care facilities, three specialized facilities for frail people and a prison's medical department [11].

The asynchronous dental tele-consultation process is composed of the following three steps (Figure 1):

$1^{\text {st }}$ step

The nurse takes pictures and videos of the patient's mouth and teeth with a specific tool: the Soprocare $\AA$ intraoral camera that reveals, using a unique fluorescence technology, dental caries, new and old dental plaque and gingival inflammation without the need for dye solutions. The camera is connected to a laptop which runs the e-DENT software and in which the nurse creates the patient's file and saves the collected images and videos.

$2^{\text {nd }}$ step

As soon as the laptop is connected to the internet, the collected data is sent to the server hosted by the Montpellier University Hospital (France).

$3^{\text {rd }}$ step

The tele-consultation process is finalized by the hospital's dentist who puts a diagnosis and establishes a treatment sheet by accessing the patient's data through a web browser. He or she evaluates the dental emergency level and proposes the type of dentist or intervention needed (regular, specialist, general anesthesia)[11].

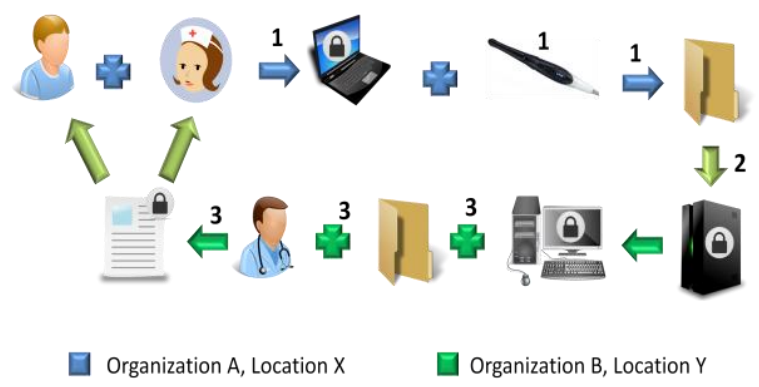

Figure 1. Oral tele-consultation process flow [11]

For the experimentation, the nurses apply the camera protocol twice for each patient with a gap of 4 to 6 months between the first and the second assessment, to compare the dental status and to measure its improvement.

The tele-consultations are carried out by dental students at the Montpellier University Hospital under the supervision and with the final approval of a licensed dentist. Patients and medical staff are informed about the established diagnosis and the required treatment.

According to the project manager, teleconsultations are preferred because they are cheaper, easier to manage and because in oral health there is no vital emergency.

\section{Assessment areas for the project}

Our team of researchers has been introduced to the specifics of the project and we were asked to identify the proper assessment/evaluation process to ensure the project's sustainability for the pilot and afterwards. We consider that the generalization of oral teleconsultations is related to the acceptance of the eDENT system, as stated by Broens [5]. Therefore the e-DENT project is currently being evaluated by a multidisciplinary team of researchers according to their field of expertise from the clinical, technology 
acceptance, medical economics and organizational point of view. In this paper, we will focus mainly on technology and procedure acceptance and the organizational changes induced by the implementation of the tele-consultation system as they are highlighted after analyzing the data collected from the patients and the nurses.

\subsection{Clinical results}

The e-DENT project aims to improve the access to dental consultations. The evaluation criteria should be the number of patients evaluated and the number of patients requiring treatment. In fact this is only one of the considered and measured variables. All proposed treatment sheets which are directly linked to the established diagnosis are compared with the actual treatments realized. This has become necessary since the dentist who sets the diagnosis and the dentist who performs the treatments are never the same and discrepancies need to be registered and investigated. The fact that treatments, which are beyond the scope of the project itself, are considered as an evaluation criteria for the project is unusual but it will become even more so for the following topics.

\subsection{Technology acceptance and tele- consultation procedure acceptance}

Semi-structured interviews are administered to all stakeholders for evaluating the acceptance of the system, starting with the nurses administering the camera protocol, the nurses who are not involved in the project, the dentists doing the diagnosis, dentists in private practice-not involved in the project, dentists treating patients who received the indication to undergo treatments after a tele-consultation, managers, directors of the facilities, health insurance and social security representatives. In this paper, we present early findings resulting from analyzing the nurses' interviews before the project and we propose a methodological approach for evaluating the cognitively impaired patients' perception of the teleconsultation procedure in which they take part.

3.2.1. Theoretical background. The interview guides are constructed around the TAM(Technology Acceptance Model) which suggests that the acceptance of a technology is influenced by the perceived usefulness and ease of use in organizational environments [7]. This model has been improved and has led to two closely related models: TAM3 [26] and Unified Theory of Acceptance and Use of Technology (UTAUT) [25]. These theories focus on cognitive variables as part of behavior change, and share the assumption that attitudes, beliefs and future outcomes are major determinants of behavior. These same theories have also been used in healthcare related behavior [18]. The patients, who are elderly, with minor, mild or severe cognitive impairment represent a challenge for this kind of assessment since the most commonly used theories related to behavior are those within the cognitive perspective [15].

In healthcare, the main concepts to address the issue of health management are compliance and adherence.

In 1976, Sackett and Haynes [13] defined compliance as "the extent to which a person's behavior coincides with the clinical prescription". Thus, healthcare professionals consider a patient compliant when the patient follows the recommendations for health care management. On the contrary, a patient is "non-compliant" when he/she does not follow the recommendations correctly.

The term "adherence" has recently replaced the term "compliance". Adherence has been defined as the "active, voluntary, and collaborative involvement of the patient in a mutually acceptable course of behavior to produce a therapeutic result" [8]. This definition implies that the patient has a choice [8]. Nieuwlaat et al. [19] have defined adherence as "the extent to which patients follow the instructions they are given for prescribed treatments". Adherence is a more neutral term than 'compliance', which can be construed as being judgmental" [19].

Compliance and Adherence are a major issue in healthcare today and there is a lot of research on adherence to medication [20]. There are many different methods for measuring adherence. Osterberg and Blaschke [20] categorized these methods as either direct or indirect. Chen \& Chan [6] consider the most recent developments in adherence research and summarize direct and indirect measurements. To assess adherence, patient interviews or self-report questionnaires are mostly used. Direct methods include direct observation by the provider.

For our study, the cognitive theories presented above (technology acceptance and adherence theory) present some limitations. According to the tenth revision of the WHO (World Health Organization), intellectual disability contributes to the overall level of intelligence, such as cognitive, language, motor and socialization functions.

The cognitive disability and impairment of our sample limits the usage of technology acceptance or adherence and compliance theories and any measurements that imply user rationality, because the subjects are not able to respond to self-reported measures or to understand the questions. There is a 
small number of researches focused on acceptance by older people in information system (Chen 2011) and even less concerning people with cognitive disability and impairment [22]. As cognitive theories could not be addressed for our study, we have decided to consult the literature review on patient observation and elderly patients to explore the acceptance issues in the healthcare context. This literature review includes observation of pain and mood or affect because these concepts are largely used to address patient observation, especially those with cognitive disability and impairment, in the healthcare context.

For the patients' observation in the healthcare field, different concepts have been used. Some researchers observe the patient's quality of life [10], others observe the patient's pain [1];[2] or patient's mood [28].

Concerning pain, a lot of measuring instruments are available [1]. The use of self-reported measures of pain (such as the visual analogue scale) is often problematic for older adults with severe cognitive impairment. Observational measures of pain have been developed to address this concern such as PACSLAC-F (Pain Assessment Checklist for Seniors with Limited Ability to Communicate)[2] or Elderly Pain Caring Assessment (EPCA)[17].

In recent research, affect and mood observation have also been used to address issues of emotional experience [28]. Watson et al. [27] developed the Positive and Negative Affect Schedule (PANAS), which consists of two 10-item scales, one for PA (Positive Affect) and one for NA (Negative Affect). These two dimensions account for three-quarters of the common variance in mood terms [29]. The PANAS scale provides humor/mood measurement through a number of words and phrases that describe different feelings and emotions.

3.2.2. Methodological approach. For the nurses, semi-structured interviews based on a previously constructed interview guide are administered.

For the patients' assessment an observation form has been created. Its purpose is to focus the observation of the patient during the procedure on two major areas: the patient's behavior and the patient's humor/mood. The specific context of the observation, the dentistry field, elderly patients with minor, mild or severe cognitive impairment led us towards the criteria included in the behavior evaluation, namely: fear of the electrical toothbrush-because of their similar aspect with the camera, tendency to bite the camera because it can hinder the image collection, difficulties in keeping the mouth open it can also hinder the image collection, opposing and cooperating during the procedure. We chose these variables following an evaluation of the main potential brakes in the application of the established protocol and the coherence with a pain evaluation scale.

For the patient's mood evaluation, a reduced and adapted scale of Positive and Negative Affect Schedule (PANAS)[28] was used. The Positive Affect (PA) Scale consists of 10 items concerning 'positive feelings', such as enthusiasm, interest and determination. The Negative Affect (NA) Scale consists of 10 items concerning 'negative' feelings, such as fear, sadness, anxiety and hostility. The observation form used in our research includes four PA variables and four NA variables.

As part of the e-DENT project, the nurse conducting the tele-consultation is also the one who fills out the observation form, in paper format, according to what she/he has or has not identified during the procedure. All paper observation forms were centralized by a researcher in an Excel database. In order to eliminate potential errors, an automated input form was created including all the rules governing the observation form's values. The resulting database has served as starting point for the data analysis made in IBM SPSS 22.0 and Excel 2007.

3.2.3. Sample. For this paper, we present the observations made on the first 101 patients out of the total 800 expected for the e-DENT project. The 101 tele-consultations were made between the 28th of August 2014 and 20th of October 2014. It is a fairly well balanced sample of 54 men and 47 women, with the patients' age ranging from 20 to 104 years old, with $51.48 \%$ - 70 years old or older. The sample includes patients from nine different sites out of the total 16 sites taking part in the pilot project, including eight long term care facilities for the elderly and one specialized facility for adults with severe intellectual, motor or somatic disability or multiple severe disabilities.

3.2.4. Data analysis. We used a combination of Excel 2007 and IBM SPSS Statistics version 22.0, which are mainly descriptive due to the rather small sample and not having other samples to compare our results with. The purpose of our analysis at this stage is to identify potential trends and potential flaws in our evaluation process and to be able to propose future improvements for better data accuracy and reliability. For the PANAS scale the Positive Affect - PA was observed through four variables and the Negative Affect - NA was also observed through four variables. For analysis purposes we added a derived variable PA-NA which for each patient is represented by the difference between the sum of the PA variables and the sum of the NA [29]. Even if the difference between the two sums does not represent a certainty for the positive or 
the negative mood of the patient, we take into consideration the descriptive value of the PA-NA for the tendency of the patient's mood during the procedure. The patients belong according to their required level of care, to one of the following three groups: autonomous (15.84\%), semi-autonomous $(34.65 \%)$ and dependent $(48.51 \%)$. The autonomous group is represented by patients who require no, or minimal intervention in their daily tasks. The semiautonomous group is represented by patients who need help in their daily tasks. The dependent group is represented by patients who require constant help 24/7 or they are immobilized. The three groups present a different percentage of psychotic patients, going from $16.33 \%$ for the dependent group, $31.43 \%$ for the semiautonomous group and up to $93.75 \%$ for the autonomous group. The high percentage of psychotic patients in the autonomous groups is explained by the fact that the majority of the patients are from a specialized facility for adults with severe intellectual, motor or somatic disability, or multiple severe disabilities.

3.2.5. Results. For the whole sample, 10 patients refused the procedure and in one additional case, this information was missing. Out of the 10 refusals, only 4 had absolutely no observation data on the observation form. According to protocol, the nurse can stop at any point if during the procedure the patient refuses to cooperate further. This is why the remaining 6 refusals that had observation data already filled in, which we consider valuable for the purpose of this paper, are part of our analysis and contribute to the results.

The derived variable PA-NA is mainly negative for the group of autonomous patients $-86.7 \%$ have a PA-NA negative or equal to 0 , so a rather negative affect during the procedure. This result could partially be explained by corroborating these findings with the fact that $93.75 \%$ of the patients in this group are psychotic. A new medical procedure, with a new tool and on top of everything an invasive one, they have to accept having something in their mouth, are reasons to believe that our findings are pertinent. Our claim is also supported by BPRS (Brief Psychiatric Rating Scale) used for establishing the severity of the psychotic disorder, which includes, among the evaluated criteria: suspiciousness- mistrust, belief others harbor malicious or discriminatory intent; hostility-animosity, contempt, belligerence, disdain for others; anxiety-worry, fear, over-concern for present or future, uneasiness; tension-physical and motor manifestations of nervousness, over-activation [21].

The derived variable PA-NA is mainly positive $61.8 \%$ for the group of semi-autonomous patients.
$31.43 \%$ of patients are psychotic and $38.2 \%$ of the patients have a PA-NA negative or equal to zero.

The derived variable PA-NA is mainly negative for the group of dependent patients, has in more than $62.5 \%$ of cases a PA-NA negative or equal to zero. Taking into consideration the profile of the patients in this group and that "dependent" implies help 24/7, the results are not surprising. Adding a new procedure in their daily routine makes them uncomfortable.

The patients throughout the whole sample have been rather cooperating than opposing during the procedure, for each of the three groups, the cooperating variable is more important than the opposing variable. The autonomous (2.86 vs.1.67) and semi-autonomous (2.64 vs. 1.88) groups present close to one whole point of difference between the averages of the two variables. The dependent (2.00 vs. 1.70) group presents only 0.3 of difference in average but still rather conflicting with the previous findings based on the PA-NA variable.

The patients have a rather equal tendency to bite the camera during the procedure with a slightly higher tendency noted for the dependent group, but remains between "a little" and "moderately".

The patients have moderate difficulty keeping their mouth open. The difficulties in keeping the mouth open are especially noted in the autonomous (3.14) group, which has a high rate of psychotic patients, so it is rather expected. For the semi-autonomous (2.28) and dependent (2.78) groups the difficulty is rather moderate.

3.2.6. Discussion. First, the data analysis shows that the majority of patients experience a negative mood during the procedure. This outcome may be related to one main concept present in the TAM3 model, namely computer anxiety. Computer anxiety consists in "the degree of an individual's apprehension, or even fear, when she/he is faced with the possibility of using computers." [24] (p. 349). Nevertheless, as in all the TAM or UTAUT concepts, computer anxiety consists in a perception and is measured by the individuals' assessment on a short scale (e.g. 3 items in the scale)[23]. We suggest that the PANAS grid may asses the anxiety in more depth, which is a key issue especially for the elderly, because they may have higher technology anxiety than younger individuals [9]; [14]. Research focusing on the acceptance of technology by the elderly is naming technology anxiety and dispositional resistance to change [12] as key concepts. These are partially included in our approach: anxiety observed as being part of the Negative Affect scale and the dispositional resistance to change, part of the behavior observation scale, "opposing". 
Second, it is important to underline that anxiety in this case is not related specifically to computers but to the tele-consultation procedure. This means that tooth brush phobia, camera fear and dentist fear can play an important role in the overall experience of the patient. Dental fear is highly specific for the dentistry field. It is named dental phobia, odontophobia, dental anxiety or, more recently, posttraumatic dental-care anxiety [4] which is described as the fear of dentistry or of receiving dental care. In France, according to an IPSOS survey realized in $2012-13 \%$ of people fear going to the dentist, with the percentage reaching $15 \%$ for Europe. In light of this, our results could be interpreted in a totally different manner, so further research and a more important sample is required.

The ageing trends in the population and the accelerated evolution of technology in all domains are a reality. The elderly have an unusual relationship with technology because they have witnessed the exponential evolution of technology but they made choices whether to use those technologies or not. Healthcare is the one domain where the elderly do not have the option to choose the procedures they undergo and the associated technology. We think that an interest towards the acceptance of the elderly is required. Specifically in the healthcare systems in which the patient is becoming an active stakeholder and he/she has to be treated accordingly. Healthcare is only the first step towards realizing that the elderly are an increasing part of the population and if today, products and services are especially designed for them; it is only a question of time before information systems in all domains also need to consider them.

3.2.7. Limitations and future perspectives. In this paper, we argue the need to explore a new methodology in order to assess the patients' acceptance of the procedures they are subjected to, when these patients are elderly and/or cognitively impaired. This methodology is based on the observation of behavior and affect. We tested this methodology in the study of patients' acceptance of the dental (oral) tele-consultation, which can also represent an issue because it includes fear of devices and dental fear.

This study has two main limitations. First, as seen during the data collection process, the human factor, the reliability of the data depends exclusively on the professionalism and on the level of implication of the medical personnel.

Second, the same medical personnel could potentially be the source of bias in the evaluation because of their relationship with the patients. For the purpose of the paper and for the e-DENT project, which will facilitate dental consultation access for these patients, we consider our approach as being the best possible approach as patient-nurse relationship is a reality for most of the cases.

For further research, as a response to the potentially unreliable data collected from one of the sites, we suggest an improvement of the process. The observation form will become digital and it will become part of the tele-consultation software, in which once a tele-consultation is finalized the corresponding observation form will no longer be editable, in this way the nurses will fill in the observation form after each tele-consultation, increasing data accuracy and reliability.

To validate our proposal would be the next logical step. To achieve this we are envisioning two separate paths. The first, which is already underway, is to collect the data for the same 100 patients for the second tele-consultation and to evaluate the changes in-between visits. The second path would be to validate the accuracy of the nurse's evaluation in comparison with the self-reported evaluation of the patient, but used for patients who do not present cognitive impairment, the prison included in the eDENT project could be a good opportunity to put this into practice. Our proposal will also gain reliability and relevance once we have extended our research to all 800 patients estimated to participate in the e-DENT experimentation.

\subsection{Cost - effectiveness and budget impact}

The medical economics evaluation of e-DENT relies heavily on the specific problem it tries to solve. Depending on the facility type, the type of deployment and the patient profile, the budget impact will be different and the cost-effectiveness evaluation will be affected. For this paper we will not go further into details on this topic but we consider important to mention it as part of the overall design of the evaluation criteria for the project.

\subsection{Organization, coordination and patient pathways}

Organizational aspects are present in the interview guides that are administered to all the stakeholders involved in the process. After a first analysis of the nurses' first interviews ( 7 in total), interview taken before the initial training and before the implementation of the project, three aspects emerged as the most important for all the nurses interviewed in the nursing homes. The first, the procedure cannot be done for all the patients, because they are very old or cognitively impaired, which will make the task very 
difficult. The second aspect is that they do not see the usefulness of the tele-consultation if there is no treatment afterwards. The third is that the importance of oral healthcare in the context of the elderly with coexisting morbidities is well known.

The organizational impact of the system can be better understood by analyzing the patient's pathway before and during the project.

3.4.1. Patient pathway before e-DENT. This is only applied in emergency cases, when the patient claims he is in pain or if the medical personnel notice something out of the ordinary. The nurse makes an appointment to a local dentist. If the patient needs special transportation, the nurse will also arrange for an ambulance to be available for the specific date. If the patient does not need special transportation he/she needs to take a cab for which he is not going to be reimbursed. At the dentist's office, he or she is taken into the consultation room and the dentist makes an examination. It can take up to 30 minutes for the patient just to be seated, according to the nurses. If the patient is diagnosed with something minor, the dentist intervenes on the spot. In some cases the patients' claims are not substantiated or the nurse, due to lack of expertise, misjudged the situation. If the patient has something more severe, in most cases he/she is asked to return a second time for treatment. This is also the case if the patient has a heart condition, diabetes or is under anticoagulant treatment and the dentist does not have the relevant information to be able to proceed with the treatment plan.

3.4.2. Patient pathway after e-DENT. The patient is undergoing a regular oral tele-consultation realized by the nurse in the healthcare facility. Besides the captured videos, the patient's file also contains information about the co-existing pathologies and relevant patient information. A dentist, in a different healthcare facility or dental practice enters the diagnosis after evaluating the patient's videos and file. The dentist also establishes a level of dental treatment urgency and proposes a treatment plan. The patient and the nurse/medical personnel are informed about the established diagnosis and the dental treatment urgency level and treatment plan. Having this information the nurse can now make an appointment to a local dentist, but having a dental diagnosis allows the dentist to better estimate the required time for the treatments. At the dentist's office, the patient has on him the diagnosis and the information about coexisting pathologies and medication, which are key for undergoing all or some of the treatments during the first visit. Having only some of the treatments proposed in the treatment plan can also be due to differences in medical opinions among two dentists, the one doing the diagnosis and the one performing the treatments.

3.4.3. Some unexpected results. In the nurses' interviews as mentioned before, the treatment is considered as an important criterion when asked about the usefulness of the e-DENT system. Since we are in the healthcare field we were expecting to have clinic variables, but the e-DENT project is supposed to propose a solution for achieving this first, critical step towards treatment, access to dental consultation. After analyzing the nurses' interviews and several days of non-participant observation it became clear that the new proposed approach has indirectly created new forms of inter- and intra-organizational coordination which seem most important in a patient centered care approach.

The new intra-organizational coordination is represented by the fact that the nurse in charge of administering the camera protocol is considered as the "expert" to contact when the medical staff is confronted with patients with dental care problems or even oral hygiene issues.

The new inter-organizational coordination has an origin in the nurse's involvement in the outcome of the tele-consultation; they are informed about the established diagnosis and more importantly the required treatment. The nurse administering the camera protocol and the dentist doing the remote video analysis are communicating with each other, exchanging information relevant for the patient's evaluation. In addition, the dentist can sometimes provide feedback on the quality of the images or request additional footage. However, the information exchange goes beyond the perimeter of the e-DENT project because the nurse also interacts with the dentist providing the treatments. This type of interaction did exist before e-DENT but it was mostly limited to making the appointment with the dentist. The inter-organizational coordination is even more surprising when we take into consideration that dentists, in general, are more used to working on their own, to be in a way isolated from the rest of the health care professionals.

\section{Conclusions}

The tele-consultation procedure is generally accepted by the elderly patients, for only 10 cases the procedure was interrupted, the nurse not being able to finalize the image collection process. During the procedure the majority of the patients are not comfortable but nevertheless they cooperate with the 
nurse. Considering the specifics of the project: dentistry, elderly or/and psychotic patients and the use of new innovative technology, we can say that our proposed methodology for assessing the patient's acceptance of the tele-consultation procedure seems to be reliable but needs a larger sample and to be tested in other similar projects.

Regarding the organizational aspects, our analysis reveals that the proposed system, the organizational support, the nurse's profile and the nurse's implication are the main factors behind the emerged intra- and inter-organizational forms of coordination which are key for the next step after the tele-consultation, the patients' treatment. Further research is required to understand the exact role of each of the identified factors and to compare these findings with the other implementation sites.

\section{References}

[1] Aubin, M. et al. 2007. L'évaluation systématique des instruments pour mesurer la douleur chez les personnes âgées ayant des capacités réduites à communiquer. Pain Research \& Management: The Journal of the Canadian Pain Society. 12, 3 (2007), 195-203.

[2] Aubin, M. et al. 2008. Validité et utilité clinique d'une grille d'observation (PACSLAC-F) pour évaluer la douleur chez des aînés atteints de démence vivant en milieu de soins de longue durée. Canadian Journal on Aging/La Revue canadienne du vieillissement. 27, 01 (Jan. 2008), 45-55.

[3] Bellamy, V. and Beaumel, C. 2014. Bilan démographique 2013: trois mariages pour deux Pacs. Insee première. 1482, (2014).

[4] Bracha, Stefan et al. 2006. Posttraumatic Dental-Care Anxiety: Is" dental phobia" a misnomer? Hawaii Dental Journal 37(5).

[5] Broens, T.H.F. et al. 2007. Determinants of successful telemedicine implementations: a literature study. Journal of Telemedicine and Telecare. 13, 6 (Sep. 2007), 303-309.

[6] Chen, K. and Chan, A.H.S. 2011. A review of technology acceptance by older adults. Gerontechnology. 10, 1 (Jan. 2011), 1-12.

[7] Davis, F.D. et al. 1989. User acceptance of computer technology: a comparison of two theorical models. Management Science. 35, 8 (1989).

[8] Delamater, A.M. 2006. Improving Patient Adherence. Clinical Diabetes. 24, 2 (Apr. 2006), 71-77.

[9] Dyck, J.L. et al. 1998. The changing construct of computer anxiety for younger and older adults. Computers in human behavior. 14, 1 (1998), 61-77.

[10] Gerritsen, D.L. et al. 2007. Measurement of overall quality of life in nursing homes through self-report: the role of cognitive impairment. Quality of Life Research. 16, 6 (Aug. 2007), 1029-1037.
[11] Giraudeau, N. et al. Projet e-DENT : téléconsultation bucco-dentaire en EHPAD. European Research in Telemedicine / La Recherche Européenne en Télémédecine.

[12] Guo, X. et al. 2013. The dark side of elderly acceptance of preventive mobile health services in China. Electronic Markets. 23, 1 (Mar. 2013), 49-61.

[13] Haynes, R.B. and Sackett, D.L. 1976. Compliance with therapeutic regimens. Johns Hopkins University Press.

[14] Laguna, K. and Babcock, R.L. 1997. Computer anxiety in young and older adults: Implications for human-computer interactions in older populations. Computers in human behavior. 13, 3 (1997), 317-326.

[15] Leventhal, H. and Cameron, L. 1987. Behavioral theories and the problem of compliance. Patient Education and Counseling. 10, 2 (Oct. 1987), 117138.

[16] Mathers, C.D. and Loncar, D. 2005. Updated projections of global mortality and burden of disease, 2002-2030: data sources, methods and results. Geneva: World Health Organization. (2005).

[17] Morello, R. et al. 2007. A scale to measure pain in non-verbally communicating older patients: The EPCA-2: Study of its psychometric properties. Pain. 133, 1 (Dec. 2007), 87-98.

[18] Munro, S. et al. 2007. A review of health behaviour theories: how useful are these for developing interventions to promote long-term medication adherence for TB and HIV/AIDS? BMC Public Health. 7, 1 (Jun. 2007), 104.

[19] Nieuwlaat, R. et al. 2014. Interventions for enhancing medication adherence. Cochrane Database of Systematic Reviews. Issue 11, Art. No.:CD000011 (2014).

[20] Osterberg, L. and Blaschke, T. 2005. Adherence to Medication. New England Journal of Medicine. 353, 5 (Aug. 2005), 487-497.

[21] Overall, J.E. and Gorham, D.R. 1962. The brief psychiatric rating scale. Psychol.

[22] Phang, C.W. et al. 2006. Senior Citizens' Acceptance of Information Systems: A Study in the Context of eGovernment Services. IEEE Transactions on Engineering Management. 53, 4 (Nov. 2006), 555569.

[23] Thatcher, J.B. and Perrewe, P.L. 2002. An empirical examination of individual traits as antecedents to computer anxiety and computer self-efficacy. Mis Quarterly. (2002), 381-396.

[24] Venkatesh, V. 2000. Determinants of Perceived Ease of Use: Integrating Perceived Behavioral Control, Computer Anxiety and Enjoyment into the Technology Acceptance Model. Information Systems Research. 11 (2000), 342-365.

[25] Venkatesh, V. et al. 2003. User Acceptance of Information Technology: Toward A Unified View. MIS Quarterly. 27, 3 (2003), 425-478.

[26] Venkatesh, V. and Bala, H. 2008. Technology acceptance model 3 and a research agenda on interventions. Decision sciences. 39, 2 (2008), 273 315. 
[27] Watson, D. et al. 1988. Development and validation of brief measures of positive and negative affect: The PANAS scales. Journal of Personality and Social Psychology. 54, 6 (1988), 1063-1070.

[28] Watson, D. and Clark, L.A. 1997. Measurement and Mismeasurement of Mood: Recurrent and Emergent issues. Journal of Personality Assessment. 68, 2 (Apr. 1997), 267-296.

[29] Watson, D. and Clark, L.A. 1999. The PANAS-X: Manual for the positive and negative affect scheduleexpanded form.

[30] WHO | Facts about ageing: http://www.who.int/ageing/about/facts/en/. Accessed: 2015-01-11. 